\title{
Artificial Intelligence Technology for COVID-19 Detection at Early Stage with Special Reference to Health Disparity and Socioeconomic Status
}

\author{
Anita Mandal ${ }^{1}$, Noor K. Islam ${ }^{2}$, and Prabir K. Mandal ${ }^{3 *}$ \\ ${ }^{1}$ Department of General Studies, Edward Waters College, 1658 Kings Road, Jacksonville, FL 32209 \\ ${ }^{2}$ Department of Social \& Behavioral Sciences, Edward Waters College, 1658 Kings Road, Jacksonville, FL 32209 \\ ${ }^{3}$ Department of Mathematics \& Sciences, Edward Waters College, 1658 Kings Road, Jacksonville, FL 32209
}

*Corresponding Author: Prabir K. Mandal. Department of Mathematics \& Sciences, Edward Waters College, 1658 Kings Road, Jacksonville, FL 32209.

Received date: August 14, 2020; Accepted date: August 25, 2020; Published date: September 01, 2020

Citation: Anita Mandal, Noor K. Islam, and Prabir K. Mandal, Artificial Intelligence Technology for COVID-19 Detection at Early Stage with Special Reference to Health Disparity and Socioeconomic Status J, Biotechnology and Bioprocessing 1(1);DOI:10.31579/ 2766-2314/003

Copyright: (C) 2020, Prabir K. Mandal: This is an open access article distributed under the Creative Commons Attribution License, which permits unrestricted use, distribution, and reproduction in any medium, provided the original work is properly cited.

\begin{abstract}
A novel coronavirus, SARS-CoV-2, was identified as the cause of an outbreak of viral pneumonia in Wuhan, China. The disease, later named coronavirus disease 2019 (COVID-19), subsequently spread globally. COVID- 19 is an aggressive disease with a low median survival rate. Ironically, the treatment process is long and very costly due to its high recurrence and mortality rates. Accurate early diagnosis and prognosis prediction of COVID-19 are essential to enhance the patient's survival rate. Mount Sinai researchers are the first in the country to use artificial intelligence (AI) combined with imaging, and clinical data to analyze patients with COVID-19. They have developed a unique algorithm that can rapidly detect COVID-19 based on how lung disease looks in computed tomography (CT scans) of the chest, in combination with patient information including symptoms, age, bloodwork, and possible contact with someone infected with the virus. AI has huge potential for analyzing large amounts of data quickly, an attribute that can have a big impact in a situation such as a pandemic. There is increasing evidence that some racial and ethnic minority groups are being disproportionately affected by COVID-19.
\end{abstract}

Key words: Artificial Intelligence Technology; COVID-19; Socioeconomic Status

\section{Introduction}

The pandemic by COVID-19 is a live issue affecting people worldwide. The sudden explosion and uncontrolled worldwide spread of COVID-19 show the limitations of existing healthcare systems to timely handle public health emergencies. In such contexts, innovative technology such as AI have emerged as promising solution for fighting coronavirus epidemic. AI provides intelligent solutions for identifying symptoms caused by coronavirus for treatments and supporting drug manufacturing. The clinical features of COVID-19 are varied, ranging from asymptomatic state to acute respiratory distress syndrome and multi organ dysfunction. The common clinical features include fever (not in all), cough, sore throat, headache, fatigue, headache, myalgia and breathlessness. AI model is as accurate as an experienced radiologist in diagnosing the disease, and even better in some cases where there will be no clear sign of lung disease on CT. We believe that the model should be viewed as an assistant (computer-aided) rather than replacement or substitution to professional radiological practice. We keep improving the knowledge of the model performance with more data collected on daily basis as well as fine tuning the architecture of the model. AI provides intelligent solutions for identifying symptoms caused by coronavirus for treatments and supporting drug manufacturing.
As with many other historical outbreaks and health phenomena, COVID-19 is impacting victims at an unequal ratio. As the cases of COVID-19 continue to increase across the world, evidence is continuing to emerge that the pandemic could be disproportionately affecting people from black, Asian, and minority ethnic (BAME) communities. There were large disparities in the proportion of people at risk of COVID-19 from minority populations. A meta-analysis was conducted of the socioeconomic disparities affecting COVID-19 and the specific barriers contributing to the high level of disparity. Literature revealed that lower socio-economic areas present challenges to practicing COVID-19 safety behaviors that may not exist in areas of higher socio-economic status. These include type of job, mode of transportation, structure/type of housing, ability to quarantine, education, income, wealth gaps and access to health resources. These barriers may prevent individuals from following safety recommendations and may account for increased cases or deaths from COVID-19 in lower socioeconomic areas. Black Americans represent $13.4 \%$ of the American population, according to the US Census Bureau, but counties with higher black populations account for more than half of all Covid-19 cases and almost $60 \%$ of deaths. Coronaviruses are large (longest genome of any RNA virus), enveloped RNA viruses of both medical and veterinary importance. The nature of the coronavirus genome is non-segmented, single stranded, and positivesense RNA. Its size ranges from $27-32 \mathrm{~kb}$, which is significantly larger 
when compared with other RNA viruses. The gene encoding the large surface glycoprotein. The research explores the impact of socioeconomic factors on COVID-19 behaviors and health outcomes. The anti-viral immune response is crucial to eliminate the invading virus, but a robust and persistent anti-viral immune response might also cause massive production of inflammatory cytokines and damage to host tissues. The overproduction of cytokines caused by aberrant immune activation is known as a cytokine storm. In fact, in the late stages of coronavirus disease, including SARS, MERS, and COVID-19, cytokine storms are a major cause of disease progression and eventual death. Scientists have conducted a first-in-human phase 1 clinical trial in healthy adults to evaluate the safety and immunogenicity of mRNA-1273.

The Mount Sinai team integrated data from those CT scans with the clinical information to develop an AI algorithm. It mimics the workflow a physician uses to diagnose COVID-19 and gives a final prediction of positive or negative diagnosis. The AI model produces separate probabilities of being COVID-19-positive based on CT images, clinical data, and both combined. In the event of a pandemic like COVID19 , this solution would help manage the accelerating demand from those communicable diseases. Radiologists can also achieve productivity gains working with AI from anywhere with features-rich cloud-based secure system, enables them to see more patients, interpret more images who in turn, the patients, receive more timely and accurate care.

The algorithm was shown to have statistically significantly higher sensitivity (84 percent) compared to 75 percent for radiologists evaluating the images and clinical data. The AI system also improved the detection of COVID-19-positive patients who had negative CT scans. Specifically, it recognized 68 percent of COVID-19-positive cases, whereas radiologists interpreted all these cases as negative due to the negative CT appearance. Improved detection is particularly important to keep patients isolated if scans don't show lung disease when patients first present symptoms (since the previous study showed that lung disease doesn't always show up on CT in the first few days) and COVID-19 symptoms are often nonspecific, resembling a flu or common cold, so it can be difficult to diagnose. This is important because it shows that an artificial intelligence algorithm can be trained to help with early identification of COVID-19, and this can be used in the clinical setting to triage or prioritize the evaluation of sick patients early in their admission to the emergency room.

Imaging can help give a rapid and accurate diagnosis. The lab tests can take up to two days, and there is the possibility of false negatives meaning imaging can help isolate patients immediately. The high sensitivity of AI model can provide 'a second opinion' to physicians in cases where CT is either negative (in the early course of infection) or shows nonspecific findings, which can be common. It is something that should be considered on a wider scale. This is an early proof concept that we can apply to our own patient data to further develop algorithms that are more specific to our southern region and diverse especially minority populations.

AI can be used to detect virus and predict how the virus is going to spread by analyzing the combined information of environmental conditions, access to healthcare, and the way it is transmitted. Based on that, AI can identify coronavirus within localized outbreaks of the disease and help reveal the nature of the virus. The coronavirus can cause severe symptoms such as pneumonia, severe acute respiratory syndrome, and kidney failure. AI-based algorithms such as genome-based neural networks already built for personalized treatment can prove very useful in controlling these adverse events or symptoms caused by a coronavirus, especially when the impact of the virus depends on immunity and the genome structure of individuals and no standard treatment can treat all symptoms effectively at present. Further, the use of AI may be very helpful in identifying the relationship of novel coronavirus and related viruses such as SARS to accelerate the finding of a new vaccine.

\section{Discussion}

The ongoing economic and social impact of the pandemic is staggering, but despite a daily flood of news on the disease, few laypeople know that paradoxically, COVID-19 mostly kills through an overreaction of the immune system. A COVID-19 vaccine is a biotechnology product intended to provide acquired immunity against COVID-19. As of August 2020 , there were 231 vaccine candidates in development, although no candidate has completed clinical trials to prove its safety and efficacy. In August, at least 24 vaccine candidates were announced or undergoing clinical trials, with six beginning Phase III and 18 still in Phase I-II. Without fundamental therapeutic interventions, current management is to reduce the virus spread and provide supportive care for diseased patients. There is an urgent need to develop targeted therapies.

\section{References}

1. Zhu, N. et al. (2020). A novel coronavirus from patients with pneumonia in China, 2019. N. Engl. J. Med. 382, 727-733.

2. Tan, W. et al. (2020). A novel coronavirus genome identified in a cluster of pneumonia cases-Wuhan, China 2019-2020. China CDC Weekly 2, 61-62.

3. Chan, J. F. et al. (2020). A familial cluster of pneumonia associated with the 2019 novel coronavirus indicating person-to-person transmission: a study of a family cluster. Lancet. 395, 514-523.

4. Huang, C. et al. (2020). Clinical features of patients infected with 2019 novel coronavirus in Wuhan, China. Lancet 395, 497-506.

5. Phan, L. T. et al. (2020). Importation and human-to-human transmission of a novel coronavirus in Vietnam. N. Engl. J. Med. $382,872-874$.

6. Li, Q. et al. (2020). Early transmission dynamics in Wuhan, China, of novel coronavirus-infected pneumonia. N. Engl. J. Med. 382, 1199-1207.

7. Chung, M. et al. (2020). CT imaging features of 2019 novel coronavirus (2019-nCoV). Radiology 295, 202-207.

8. Phelan, A. L., Katz, R. \& Gostin, L. O. (2020). The novel coronavirus originating in Wuhan, China: challenges for global health governance. JAMA 323, 709-710.

9. Nishiura, H. et al. (2020). The extent of transmission of novel coronavirus in Wuhan, China, 2020. J. Clin. Med. 9, 330.

10. Wang, D. et al. (2020). Clinical characteristics of 138 hospitalized patients with 2019 novel coronavirus-infected pneumonia in Wuhan, China. JAMA 323, 1061-1069.

11. Kanne, J. P. \& Chest, C. T. (2020). Findings in 2019 novel coronavirus (2019-nCoV) infections from Wuhan, China: key points for the radiologist. Radiology 295, 16-17.

12. Song, F. et al. (2020). Emerging 2019 novel coronavirus (2019nCoV) pneumonia. Radiology 295, 210-217.

13. Florida Department of Health, Division of Disease Control and Health Protection. Florida's Covid-19 Data and Surveillance Dashboard (2020).

14. Centers for Disease Control and Prevention. Coronavirus Diseases 2020 (Covid-19).

15. Galvin, G. (2020). Language Access Issues a Barrier During Covid19. U.S. News. 